\title{
Weissella confusa: problems with identification of an opportunistic pathogen that has been found in fermented foods and proposed as a probiotic
}

\author{
Marilynn R. Fairfax ${ }^{1,2 *}$, Paul R. Lephart ${ }^{2}$ and Hossein Salimnia ${ }^{1,2}$ \\ 1 Department of Pathology, Wayne State University School of Medicine, Detroit, MI, USA \\ ${ }^{2}$ Clinical Microbiology Division, Detroit Medical Center University Laboratories, Detroit, MI, USA
}

Edited by:

Vincenzina Fusco, National Research

Council of Italy, Italy

Reviewed by:

Carmen Wacher, Universidad

Nacional Autónoma de México,

Mexico

Giuseppe Blaiotta, Dipartimento di

Agraria, Italy

*Correspondence:

Marilynn R. Fairfax, Clinical

Microbiology Division, Detroit

Medical Center, University

Laboratories, 4101 St. Antoine,

Detroit, MI 48235, USA

e-mail:mfairfax@dmc.org
Weissella confusa is found in fermented foods and has been suggested as a probiotic, but also causes sepsis and other serious infections in humans and animals. The incidence of human infections is underestimated partly due to confusion with viridans streptococci and partly due to difficulty making a definitive identification, even if the organism is recognized to belong to another genus, owing to the inability of commercial organism systems to identify it. We report our experiences identifying $W$. confusa isolated from two immune-compromised patients, both of whom developed sepsis with this organism. Two MicroScan gram positive combination panels, could not identify the organism because they did not have $W$. confusa in their data bases, but did not provide a false identification. Other laboratorians have reported failure to identify or false identifications of $W$. confusa with other commercial systems. $W$. confusa is in the data base of the RapIDTM Str panel (Remel), which gave three incorrect, high probability results ( $\geq 95 \%)$. 16S rDNA sequencing identified the isolates as $W$. confusa. Maldi-Tof, performed by two of our reference laboratories, also correctly identified both isolates. Use of $W$. confusa as a probiotic should be approached with caution because its true incidence as an opportunisitic pathogen is unknown.

Keywords: Weissella confusa bacteremia, Weissella confusa identification, MALDI-TOF mass spectrometry, 16S rDNA sequence, Weissella confusa susceptibiliy testing

\section{INTRODUCTION}

A search in the PubMed data base for "Weissella confusa," performed on 24 June, 2013, revealed 74 articles about this organism. Forty of them highlighted the finding of W. confusa in fermented food, not including the initial paper by Collins et al. (1993) who constructed the genus Weissella to include some organisms found in fermented food sources. Others discussed its potential as a probiotic (for example: Aveni et al., 2001; Lee et al., 2012). Two species have been associated with human bacteremia: W. confusa (formerly Lactobacillus confusus) and W. cibaria. W. confusa infection is rare which may be due, in part, to the inability of commercial systems to identify the organism. However there were 10 papers highlighting its role as a cause of human sepsis and bacteremia (Green et al., 1990; Olano et al., 2001; Bjorkroth et al., 2002; Flaherty et al., 2007; Salimnia et al., 2010; Shin et al., 2007; Harlan et al., 2011; Kumar et al., 2011; Lee et al., 2011; Fairfax and Salimnia, 2012). These are mostly individual case reports from immune-compromised hosts. However, one paper from Taiwan reports a series of 10 bacteremias over a 10 -year period (Lee et al., 2011). About half of these were mixed infections in which the role of the $W$. confusa was undeterminable. Many of the patients died, but whether the outcome could be attributed directly to the $W$. confusa is also uncertain.

We discussed our two W. confusa isolates from septic patients in two previous papers. The first highlights the isolation of the organism from two immune-compromised patients (Salimnia et al., 2010). The second (Fairfax and Salimnia, 2012) emphasizes that the organism is probably under-reported because, by spot testing, it resembles a viridans streptococcus (Ruoff, 2002) and therefore may be reported as a skin contaminant and not worked up (Hall and Lyman, 2006). However, this is not the only problem with the identification of W. confusa. Many commercial organism identification systems fail to identify or mis-identify the organism (Salimnia et al., 2010; Lee et al., 2011; Fairfax and Salimnia, 2012). For an organism that is a candidate for inclusion in probiotic regimens, this may be dangerous, as its true pathogenic potential may not be appreciated. Our objectives with this paper are to highlight our difficulties with the organism identification in a clinical laboratory, to describe their possible resolutions, and to emphasize the importance of delineating the role of this opportunistic pathogen before deciding that it might be used as a probiotic.

\section{CASE REPORTS}

\section{PATIENT 1}

A 34-year-old man received a successful allogeneic, unrelated hematopoetic stem cell transplant for acute lymphocytic leukemia and was discharged from the hospital. On day 23 post transplant, he returned with fever, neutropenia, and graft failure. One blood culture set was drawn. Empiric therapy with vancomycin 
(VAN) and aztreonam was begun. Both the aerobic and anaerobic bottles grew gram-positive cocci/coccobacilli in pairs and chains. Although initially presumed to be a viridans streptococcus because of its alpha-hemolytic colonies and spot testing results (Ruoff, 2002) and a blood culture contaminant because it grew in only one culture set (Hall and Lyman, 2006), the organism was worked up at physician request. Two additional blood cultures, drawn when the first set turned positive, grew the same organism. The MicroScan (MS; Siemens Healthcare Diagnostics, Sacramento, CA, USA) PC20 panel showed the organism to be resistant (R) to VAN, metronidazole, and rifampin, and susceptible $(S)$ to other antibiotics on the panel appropriate for the treatment of gram positive cocci. However, it gave no identification. Etest (AB Biodisk, Solna SW) confirmed the VAN resistance and revealed that the organism was $S$ to daptomycin (DAP). DAP therapy was begun and follow up cultures were negative.

\section{PATIENT 2}

A 58-year-old man with 2nd and 3rd degree burns received extensive debridement, multiple skin grafts, a tracheotomy and prolonged mechanical ventilation. Several bouts of polymicrobial bacteremia were treated with multiple courses of antibiotics including VAN. On hospital day 29, he again developed bacteremia with both E. faecalis and a catalase-negative, grampositive coccobacillus that grew as pairs and chains both aerobically and anaerobically. Identification and susceptibility testing were similar to those for Patient 1 . His bacteremia resolved after treatment with DAP.

\section{MATERIALS AND METHODS BLOOD CULTURES}

All blood culture bottles were incubated in the Bactec 9240 blood culture system (Becton Dickinson, Sparks, MD, USA) according to the manufacturer's instructions.

\section{SPOT TESTS}

The following spot tests were performed according to the manfacturers' instructions: leucine amino peptidase (LAP Disk, Remel, Lenexa, KS, USA), pyrrolidinyl aralamidase (PYR, Identicult ${ }^{\circledR}{ }_{-}$ AE-PYR; PML Microbiologicals, Tualitin OR, USA).

\section{COMMERCIAL IDENTIFICATION AND SUSCEPTIBILITY TESTING}

The first organisms was tested on the MS PC20 panel. The second organism was inoculated into the MS PC29 Panel to which our laboratory had switched in the interim. After the MicroScan failed to identify the organisms, we used the RapID ${ }^{\mathrm{TM}}$ Str (RapID, Remel, Lenexa, Kansas, USA), according to the manufacturers' instructions, to identify the organism. $W$. confusa is in the data base of the panel.

E-tests for VAN and DAP (A B Biodisc, Solna, SW) were performed according to the manufacturers instructions. The VAN and DAP minimal inhibitory concentrations were $\geq 256 \mu \mathrm{gm}$ $\mathrm{ml}^{-1}$ and $0.5 \mu \mathrm{gm} \mathrm{ml}^{-1}$ respectively.

\section{S rDNA SEOUENCING}

$16 \mathrm{~S}$ rDNA sequencing was performed using the MicroSeq Full Gene 16S rDNA Bacterial Identification Kit (Applied Biosystems).
BLAST analysis was used to identify the organism (Nucleotide collection (nr/nt) database, at the NCBI website; http://blast.ncbi. nlm.nih.gov/Blast.cgi).

\section{RESULTS}

The MS gram-positive panels gave antibiotic susceptibility results but failed to identify the organism. Several attempts at organism identification using RapID gave three incorrect identifications depending on the interpretation of ambiguous reactions, all at probabilities greater than 95\%: Streptococcus intermedius, Listeria monocytogenes, Pediococcus pentosaceus (Table 1). On RapID, W. confusa should have been positive for arginine (ARG) and esculin (ESC) hydrolysis, and given negative reactions for all other biochemicals (Table 1). The panel was run twice, on isolates from different days, by different experienced technologists. The first time the ARG, ESC, p-nitro-phenyl- $\alpha, \mathrm{D}$-glucoside (GLU), glucosaminide (NAG) and p-nitrophenyl phosphate (PO4) were positive. This yielded an identification of Streptococcus intermedius with a probability of $95.8 \%$, although the web site cautioned that lysine-negative isolates were uncommon. The second time, ARG and PO4 hydrolysis were negative and GLU and NAG were ambiguous. If GLU and NAG were interpreted as positive, the isolate should have been Listeria monocytogenes. If both were negative, it would have coded as Pediococcus pentosaceus. These organisms were eliminated by colony and/or gram stain morphology, and by the results from the MS panels. The organism was subjected to $\mathrm{rDNA}$ sequencing.

The sequence obtained by $16 \mathrm{~S}$ rDNA sequencing had $100 \%$ identity to $W$. confusa strain Inje LM S-338 (DQ321751.1) with no gaps and a maximum score of 2754 .

The organism from the second patient was similar in colony and gram stain morphology and in spot test results to the isolate from patient 1 . However it was not assumed to be a contaminant as it grew in both bottles of two different blood cultures. It was analyzed on the Microscan MS PC29 panel which also did not identify the isolate. The biochemical and antibiotic susceptibility results were similar to those of the first isolate, except for a positive Voges-Proskauer (VP, acetoin) reaction. The W. confusa isolate reported by Olano et al. (2001), and those in references cited by them, gave negative VP reactions. It was also sent for $16 \mathrm{~S} \mathrm{rDNA}$ analysis and the sequence was $100 \%$ identical to the first patient's isolate. It was then inoculated into the RapID panel, which again failed to support an identification as W. confusa (Table 1).

\section{DISCUSSION}

Weissella confusa is appropriately named. Difficulty identifying it by standard clinical laboratory techniques may contribute to an underappreciation of its role as an opportunistic pathogen. This is highlighted by our experience with two cases of $W$. confusa causing serious infections in immune compromised patients. Initially thought to be viridans streptococci, and skin contaminants because they were catalase and PYR negative and LAP positive (Ruoff, 2002), the isolates from our two immune-compromised patients might have been dismissed as contaminants if the physician for the first had not requested identification and if the second had not grown in multiple blood cultures sets (Hall and Lyman, 2006). Commercial organism identification and susceptibility 
Table 1 | Biochemical reactions of Weissella confusa isolates from Patients 1 and 2 compared with identification criteria from commercial identification systems.

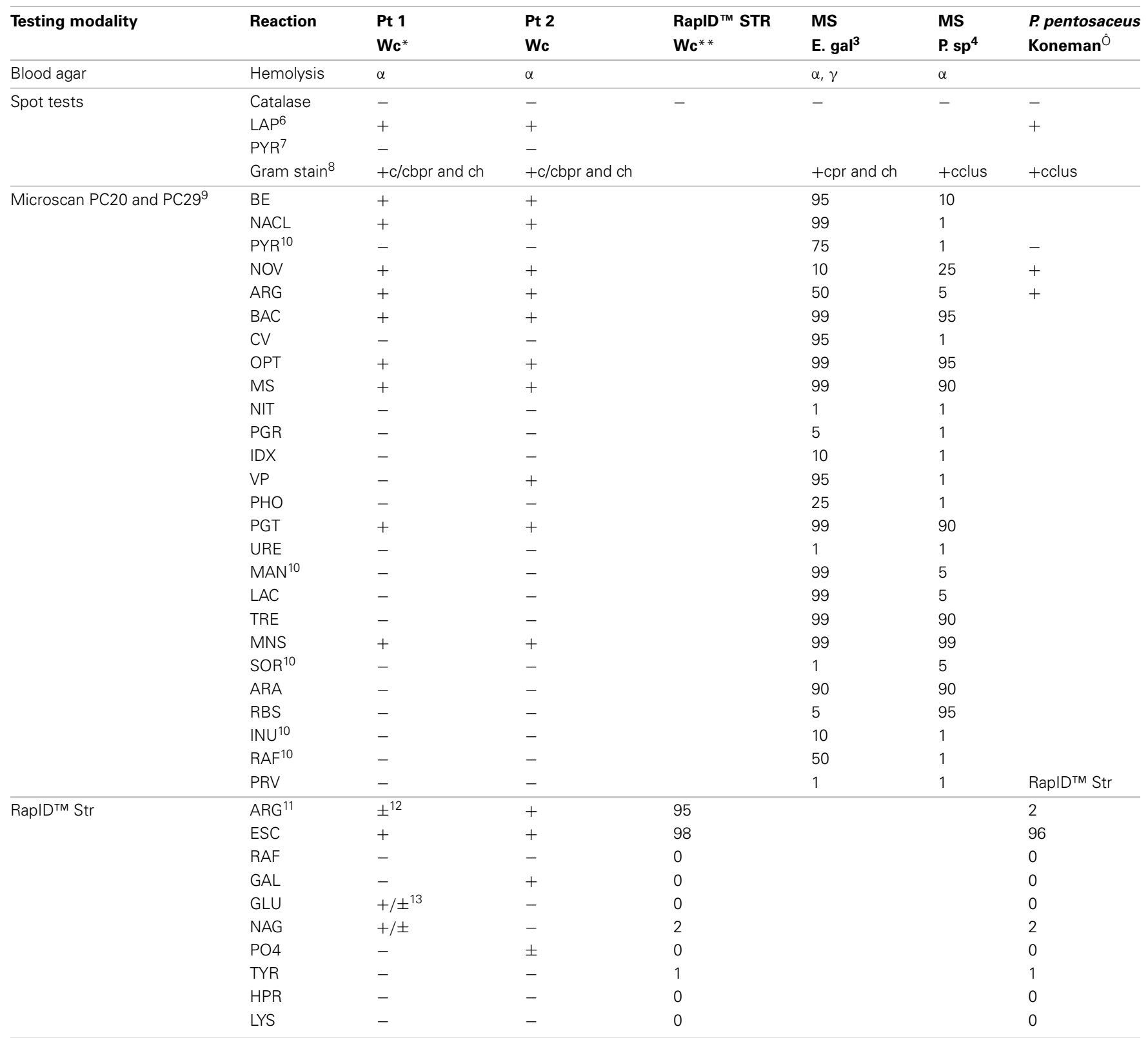

${ }^{*}$ We: Weissella confusa.

${ }^{* *} W$. confusa biochemical reactions according to the package insert with the RapIDTM STR panel.

${ }^{3}$ E. gal: Enterococcus gallinarum biochemical reactions as shown in MicroScan literature.

${ }^{4}$ Pediococcus species biochemical reactions as shown in MicroScan literature.

Ô P. pentosaceus biochemical reactions from Winn et al. (2006).

${ }^{6}$ LAP, leucine aminopeptidase.

${ }^{7}$ PYR, pyrrolidinyl arylamidase.

${ }^{8}$ Abbreviations for the gram stain interpretation are: $c$, cocci; cb, coccobacilli; pr, pairs; ch, chains; clus, clusters.

${ }^{9}$ The reagent names on the MicroScan panels are as follows: BE, 40\% bile esculin; NACL, 6.5\% sodium chloride; PYR, L-pyrrolidonyl- $\beta$-naphthylamide; NOV, novobiocin, ARG, arginine; BAC, bacitracin; CV, crystal violet; OPT, optochin; MS, Micrococcus screen, NIT, nitrate; PGR, PNP- $\beta$-D-glucuronide; IDX, indoxyl phosphatase; VP, VogesProskauer; PGT, PNP- $\beta$-D-galactopyranoside, URE, urea; MAN, mannitol; LAC, lactose; TRE, trehalose; MNS, mannose; SOR, sorbitol; ARA, arabinose, RBS, ribose; INU, Inulin; PRV, pyruvate. All the biochemical reagents from the Microscan PC20 and 29 panels are included here, but they have been reordered to place those that we consider most important for Weissella confusa identification at the top of the column.

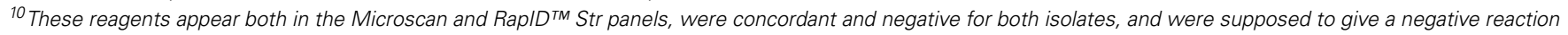

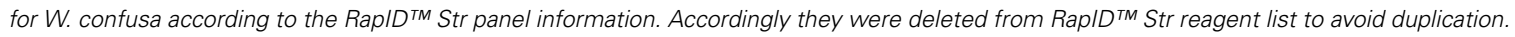

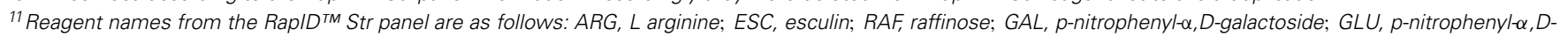
glucoside; $\mathrm{PO}_{4}$, p-nitrophenyl phosphate; TYR, tyrosine $\beta$-naphthylamide; HPR, hydroxyproline $\beta$-naphthylamide; LYS, lysine $\beta$-naphthylamide. See also footnote 10.

${ }^{12}$ Different reactions from two different blood culture isolates from patient one, drawn 2 days apart.

${ }^{13} \pm$ Ambiguous reaction requiring subjective interpretation. 
testing systems play increasing roles in US microbiology, and it is important that they provide accurate identification or indicate that the organism cannot be identified by their system.

W. confusa is not in the MicroScan data base and their PC20 and PC29 panels did not provide an identification, but the biochemical reactions pointed in the correct direction. W. confusa is in the data base of the RapID panel, but it provided three incorrect, high-probability identifications on the isolates from the first patient and failed to confirm the identity of the second (Table 1). $16 S$ rDNA sequencing identified $W$. confusa.

Lee et al. (2011) reported that none of their ten $W$. confusa isolates were identified, even to the species level, by the Phoenix Automated Microbiology System (Becton Dickinson) and the Vitek 2 system (BioMerieux. Marcy l'Etoile, France). The former system identified five of the isolates as Leuconostoc mesenteroides, and two as Streptococcus bovis II, and one each was identified as Lactobacillus lactis and Staphylococcus hominis. One remained unidentified. On the Vitek, four were identified as $L$. pseudo-mesenteroides, two as Pediococcus pentosaceus, and one as Steptococcus agalactiae/ equinus. Three were not identified. We believe that it is more dangerous to provide a false characterization of the organism, than to say that the organism cannot be identified. This is expecially true in cases when the actual antibiotic susceptibilities of the organism differ from those that would be associated with the organism that was erroneously identified (S. agalactiae).

Matrix-assisted laser desorption ionization time-of-flight (Maldi-ToF) is increasingly being used as an organism identification system, particularly in large microbiology laboratories, because it is fast, accurate, and generally regarded as economical, despite its high initial cost. It is less costly and less labor-intensive than 16S rDNA sequencing. We sent both isolates to two reference laboratories for identification and both were identified as W. confusa by Maldi-ToF. Furthermore, Fusco et al. (2011) have developed a PCR assay which is specific for W. confusa and could be used to unambiguously identify it in foods and clinical specimens. Thus it is hoped that future W. confusa isolates may be rapidly and accurately identified.

VAN is generally regarded as an essential component in grampositive and empiric antibiotic regimens. This is clearly not the case, however, and Leuconostoc, Lactobacillus, Pediococcus, some Enterococcus sp. as well as $W$. confusa have been reported to be intrinsically R to VAN (see Lee et al., 2012). Our two isolates were also R to metronidazole. They were susceptible to all other antibiotics that are generally regarded as suitable for gram-positive cocci. However, Lee et al. (2012) reported that their 10 isolates were all resistant to trimethoprim-sulfamethoxazole (MIC $\geq$ $128 \mathrm{\mu gm} \mathrm{ml}^{-1}$ ).

In conclusion, although $W$. confusa is a known opportunistic pathogen, it is difficult to identify in the clinical laboratory. It appears to be relatively common in fermented foods, and has been proposed as a probiotic although it is intrinsically resistant to vancomycin. It may be selected for in those who have received vancomycin therapy, as did our second patient presented above. Then it may be identified as a viridans Streptococcus because of its colony morphology, gram-stain morphology, and spot testing results, and not fully worked up or treated, as was almost the case with our first patient. Furthermore, even if it is recognized as the etiologic agent of disease, it is either not identified or mis-identified by major commercial organism identification systems that are used in clinical microbiology laboratories. Thus the incidence of disease caused by $W$. confusa is likely to be seriously underappreciated, and its inclusion in probiotic regimens should proceed with caution. Maldi-ToF is becoming more common in clinical laboratories throughout the world, and it is hoped that, if $W$. confusa is in the data base, the full spectrum of disease caused by this organism will be better recognized.

\section{AUTHOR CONTRIBUTIONS}

All authors contributed to the acquisition and interpretation of data, the conception of the work, the drafting and revision of the manuscript and of its final approval.

\section{ACKNOWLEDGMENTS}

We thank Christine Wollenweber and Robert Mitchell for their assistance.

\section{AUTHOR NOTE}

Some of this information was presented at the Combined Meeting of the International Congress on Antimicrobial Agents and Chemotherapy and the Infectious Diseases Society of America, Chicago, IL, USA in October 2008 (abstract number D-4004), It was also published as a case report (Salimnia et al., 2010) and as one of the cases in a review article about unusual etiologic agents of sepsis that can appear to be blood culture contaminants (Fairfax and Salimnia, 2012).

\section{REFERENCES}

Aveni, F. A., Sanchez, B., Adenivi, B. A., de Los Reyes-Gavilan, C. G., Margolles, A., and Ruas-Madiedo, P. (2001). Evaluation of the functional potential of Weissella and Lactobacillus isolates obtained from nigerian traditional fermented foods and cow's intestine. Int. J. Food Microbiol. 147, 97-104. doi: 10.1016/j.ijfoodmicro.2011.03.014

Bjorkroth, K. J., Schillinger, U., Geisen, R., Weiss, N., Hoste, B., Holzapfel, W. H., et al. (2002). Taxonomic study of Weissella confusa and description of Weissella cibaria sp. nov., detected in food and clinical samples. Int. J. Syst. Evol. Microbiol. 52, 141-148.

Collins, S. J., Metaxopoulus, J., and Wallbnks, S. (1993). Taxonomic studies on some Leuconostoc-like organisms from fermented sausages: description of a new genus Weissella for the Leuconostoc paramesenteroides group of species. J. Appl. Bacteriol. 75, 595-603. doi: 10.1111/j.1365-2672.1993.tb01600.x

Fairfax, M., and Salimnia, H. R. (2012). "Beware of unusual organisms masquerading as skin contaminants," in Sepsis-an Ongoing and Significant Challenge, ed L. Azevedo (Rijeka: Intech Open), 275-286.

Flaherty, J. D., Levett, P. N., Dewhirst, F. E., Troe, T. E., Warren, J. R., and Johnson, S. (2007). Fatal case of endocarditis due to Weissella confusa. J. Clin. Microbiol. 41, 2237-2239. doi: 10.1128/JCM.41.5.2237-2239.2003

Fusco, V., Quero, G. M., Stea, G., Morea, M., and Visconti, A. (2011). Novel PCRbased identification of Weissella confusa using an AFLP-derived marker. Int. J. Food Microbiol. 145, 437-443. doi: 10.1016/j.ijfoodmicro.2011.01.015

Green, M., Wadowsky, R. M., and Barbadora, K. (1990). Recovery of vancomycinresistant gram-positive cocci from children. J. Clin. Microbiol. 28, 484-488.

Hall, K. K., and Lyman, J. A. (2006). Updated review of blood culture contamination. Clin. Microbiol. Rev. 19, 788-802. doi: 10.1128/CMR.00062-05

Harlan, N. P., Kempker, R. R., Parekh, S. M., Burd, E. M., and Kuhar, D. T. (2011). Weissella confuse bacteremia in a liver transplant patient with hepatic artery thrombosis. Transpl. Infect. Dis. 13, 290-293. doi: 10.1111/j.13993062.2010.00579.x 
Kumar, A., Augustine, D., Sudhindran, S., Kurian, A. M., Disesh, K. R., Karim, S., et al. (2011). Weissella confusa: a rare cause of vancomycin-resistant gram-positive bacteraemia. J. Med. Microbiol. 60, 1539-1541. doi: 10.1099/jmm. 0.027169-0

Lee, K. W., Park, J. Y., Jeong, H. R., Heo, H. J., Han, N. S., and Kim, J. H. (2012). Probiotic properties of Weissella strains isolated from human faeces. Anaerobe 18, 96-102. doi: 10.1016/j.anaerobe.2011.12.015

Lee, M. R., Huang, Y. T., Liao, C. H., Lai, C. C., and Lee, P. I. (2011). Hsueh PR. Bacteraemia caused by Weissella confuse at a university hospital in Taiwan: 1997-2007. Clin. Microbiol. Infect. 17, 1226-1231. doi: 10.1111/j.14690691.2010.03388.x

Olano, A., Chua, J., Schroeder, S., Minari, A., Lasalvia, M., and Hall, B. (2001). Weissella confusa (basonym: Lactobacillus confuses) bacteremia: a case report. J. Clin. Microbiol. 39, 1604-1607. doi: 10.1128/JCM.39.4.1604-1607.2001

Ruoff, K. L. (2002). Miscellaneous catalase-negative, gram-positive cocci: emerging opportunists. J. Clin. Microbiol. 40, 1129-1133. doi: 10.1128/JCM.40.4.11291133.2002

Salimnia, H., Alangaden, G. J., Bharadwaj, R., Painter, T. M., Chandrasekar, P. H., and Fairfax, M. R. (2010). Weissella confusa: an unexpected cause of vancomycin-resistant gram-positive bacteremia in immune compromised hosts. Transpl. Infect. Dis. 12, 526-528. doi: 10.1111/j.1399-3062.2010.00539.x

Shin, J. H., Kim, D. I., Kim, H. R., Kim, D. S., Kook, J. K., and Lee, J. N. (2007). Severe infective endocarditis of native valves caused by Weissella confuse detected incidentally on echocardiography. J. Infect. 54, e149-e151. doi: 10.1016/j.jinf.2006.09.009
Winn, W., Allen, S., Janda, W., Koneman, E., Procop, G., Schreckenberger, P., et al. (2006). Koneman's Color Atlas and Textbook of Diagnostic Microbiology, 6th Edn. Baltimore, MD: Lippincott, Williams and Wilkins.

Conflict of Interest Statement: Dr. Salimnia has received research funding for unrelated projects from BioFire (Salt Lake City, UT, USA) and AdvanDx (Woburn, MA, USA). Dr. Lephart and Dr. Fairfax declare that the research was conducted in the absence of any commercial or financial relationships that could be construed as a potential conflict of interest.

Received: 23 March 2014; paper pending published: 15 April 2014; accepted: 09 May 2014; published online: 12 June 2014.

Citation: Fairfax MR, Lephart PR and Salimnia H (2014) Weissella confusa: problems with identification of an opportunistic pathogen that has been found in fermented foods and proposed as a probiotic. Front. Microbiol. 5:254. doi: 10.3389/fmicb. 2014.00254

This article was submitted to Food Microbiology, a section of the journal Frontiers in Microbiology.

Copyright (c) 2014 Fairfax, Lephart and Salimnia. This is an open-access article distributed under the terms of the Creative Commons Attribution License (CC BY). The use, distribution or reproduction in other forums is permitted, provided the original author(s) or licensor are credited and that the original publication in this journal is cited, in accordance with accepted academic practice. No use, distribution or reproduction is permitted which does not comply with these terms. 